\title{
A Robust Beat-to-Beat Artifact Detection Algorithm for Pulse Wave
}

\author{
Qihan Hu, ${ }^{1}$ Xintao Deng, ${ }^{2}$ Xin Liu, ${ }^{1}$ Aiguo Wang, ${ }^{2}$ and Cuiwei Yang $\mathbb{D}^{1,3}$ \\ ${ }^{1}$ Department of Electronic Engineering, Fudan University, Shanghai 200433, China \\ ${ }^{2}$ Department of Cardiology, Xinghua City People's Hospital, Changzhou, Jiangsu 225700, China \\ ${ }^{3}$ Key Laboratory of Medical Imaging Computing and Computer Assisted Intervention of Shanghai (200093), \\ Shanghai Engineering Research Center of Cardiac Electrophysiology, Shanghai 201318, China \\ Correspondence should be addressed to Cuiwei Yang; yangcw@fudan.edu.cn
}

Received 20 August 2020; Revised 11 October 2020; Accepted 30 October 2020; Published 12 November 2020

Academic Editor: Chenxi Huang

Copyright (c) 2020 Qihan Hu et al. This is an open access article distributed under the Creative Commons Attribution License, which permits unrestricted use, distribution, and reproduction in any medium, provided the original work is properly cited.

\begin{abstract}
With the rise of the concept of smart cities and healthcare, artificial intelligence helps people pay increasing attention to the health of themselves. People can wear a variety of wearable devices to monitor their physiological conditions. The pulse wave is a kind of physiological signal which is widely applied in the physiological monitoring system. However, the pulse wave is susceptible to artifacts, which prevents its popularization. In this work, we propose a novel beat-to-beat artifact detection algorithm, which performs pulse wave segmentation based on wavelet transform and then detects artifacts beat by beat based on the decision list. We verified our method on data acquired from different databases and compared with experts' annotations. The segmentation algorithm achieved an accuracy of $96.13 \%$. When it is applied to detect main peaks, the performance achieved an accuracy of $99.11 \%$. After the previous segmentation algorithm, the artifact detection algorithm can detect beat-to-beat pulse waves and artifacts with an accuracy of $98.11 \%$. The result indicated that the proposed method is robust for pulse waves of different patterns and could effectively detect the artifact without the complex algorithm. In summary, our proposed algorithm is capable of annotating pulse waves of various patterns and determining pulse wave quality. Since our method is developed and evaluated on the transmission-mode PPG data, it is more suitable for the devices and applications inside the hospitals instead of reflectance-mode PPG.
\end{abstract}

\section{Introduction}

Smart cities aim to provide citizens with quality life and services, and smart healthcare is its essential part. Pulse wave contains vital physiological information about the cardiovascular system, which is now commonly applied in smart healthcare (measuring blood oxygen, heart rate, and blood pressure). The acquisition of the above physiological parameters depends on two fiducial points (main peak and onset). However, the pulse wave, especially the photoplethysmographic (PPG) signal, is weak and susceptible to artifacts. Because the spectrum and duration of the artifact are difficult to estimate, artifacts are hard to eliminate with traditional filters, which affects the subsequent analysis. To get artifact-free data, a robust pulse wave preprocessing algorithm is needed.
In general, artifacts include technical, environmental, and biological artifacts [1]. Many artifact reduction methods introduce multichannel signals (such as the accelerometer and multiwavelength PPG) to assist artifact analysis [2,3], which hinders the prevalence of the wearable devices. Moreover, complex algorithms require huge computations, like adaptive filtering [4] and machine learning algorithms [5]. Moreover, artifact reduction methods can distort normal signals while correcting disturbed signals. Therefore, recent studies usually divided the signal into fixed-time segments and then discarded the disturbed segments as a whole after artifact detection [6], resulting in useful signals also filtered out along with artifacts. Therefore, if artifacts are detected beat by beat, the normal pulse waves can be preserved as much as possible. For this purpose, we propose a beat-to-beat artifact detection algorithm for pulse wave. 
Accurate detection of the onset, usually accompanied by peak detection, is essential for the periodic segmentation of the pulse wave. In reference [1], adaptive threshold and moving average filter are used to recognize the main peak. Han and Kim [7] apply a downward zero-crossing method to detect the main peak. Paradkar and Chowdhury [8] combine singular value decomposition and wavelet transform to detect the fiducial points. However, these methods are not robust enough for the diversity and variability of pulse waveforms.

In this paper, we propose a robust fiducial point detection algorithm based on wavelet transform and a beat-tobeat artifact detection method based on the decision list, which is verified on different public databases.

\section{Materials and Methods}

As Figure 1 shows, we first eliminate sensor-off and clipping waveforms and then perform noise suppression based on discrete wavelet transform (DWT). On this basis, a beat-tobeat segmentation method (onset detection) is proposed. Finally, we use the artifact detection algorithm for the segmented pulse wave to determine whether it is normal or not.

In this study, the artifact in the PPG signal is divided into two categories, as shown in Figure 2. The first kind, namely, the sensor-off/clipping waveforms, results from the sensor slipping off. The high-amplitude pulse suddenly appears in the PPG waveform. Besides, the clipping waveform occurs when the high-amplitude pulse exceeds the range of the analog-digital converter. The second kind results from the slide of the sensor caused by strenuous human movement. There appear the distortion of the PPG waveform and the loss of the fiducial points.

2.1. Database. Three public databases, Multiparameter Intelligent Monitoring in Intensive Care (MIMIC), Complex System Laboratory (CSL), and CapnoBase database, were used to verify the performance of the algorithm [9-11]. Table 1 shows the databases used to validate different algorithms. To prepare databases for artifact detection, we invited two doctors familiar with this field to annotate data manually. The annotation rules of the artifact were defined as (1) there is no physiological explanation for the change in signal morphology and (2) characteristic points cannot be identified clearly. The following describes the specific usage of the three databases used to validate the fiducial point and artifact detection algorithm.

Database for the fiducial point detection: we downloaded 10 minutes of data for each subject, for a total of 50 subjects, from the MIMIC database for the onset detection. Besides, we also adopted the ABP signal for verifying the robustness of the algorithm, just like the literature $[12,13]$. Similarly, two 60-minute ABP records from the Complex Systems Laboratory (CSL) database were downloaded for verifying the main peak detection algorithm. PPG signals from 33 subjects in the CapnoBase database were used to verify the main peak detection algorithm.
Database for artifact detection: since PPG signals are more susceptible to artifacts than ABP signals, we took oneminute PPG data containing artifacts for each subject, for a total of 27 subjects, from the MIMIC database. Besides, ABP signals in the CSL database contain fewer artifacts. Therefore, we took four 1-minute ABP signal segments for verifying the artifact detection algorithm.

Local Dataset: ten young subjects, wearing the PPG sensor (Fingertip Pulse Oximeter BM2000A, Berry), are required to sit still in the chairs for 30 seconds and walk around for 30 seconds to get a clean PPG signal and disrupted signal, respectively.

2.2. Detection of Onset and Main Peak. The noise suppression and onset detection pipelines are presented in Figure 3. Their specific parameter settings are shown in Figure 3(a). Since CSL and CapnoBase databases have been preprocessed, we only need to process the data from the MIMIC database. Since the sample rate of the MIMIC is $125 \mathrm{~Hz}$ and the main components of the pulse wave occur in the range of $0.5-15 \mathrm{~Hz}$ [14], a sevenlayer DWT is performed based on Daubechies $(\mathrm{Db}) 8$ wavelet. Then, the first-layer approximation subband (corresponding to low-frequency noise) and sixth to seventh detail subbands (corresponding to high-frequency noise) are discarded by zeroing their decomposition coefficients. Then, the clean signal is obtained by the reconstruction of the decomposition. As shown in Figure 4, the noise suppression algorithm eliminates baseline drift and retains the fiducial points.

After noise suppression, we detect the onset and main peak by stationary wavelet transform (SWT). The basic idea and parameter settings of this method are shown in Figure 3(b). The first stage divides the clean signal into segments and applies SWT with second-order spline wavelets, which has an excellent detection effect on singularity points. In the second to fourth stages, we define the region where the onset exists by peaks of each subband. Therefore, an adaptive threshold is calculated, which is 0.2 (empirical parameter) of the maximum value of the current subband in the current segment. Then, the local maximum is regarded as a peak in every subband above this threshold. Towering peaks in the third and fifth detail subbands define the region containing the onset (see Figure 5). However, sometimes this region does not include the onset. Therefore, we extend this region by a certain length, which is 0.5 (empirical parameter) of the average distance between the peak position of the fifth subband and the peak position of the sixth subband. Finally, we determine the location of the minimum in the expanded region as the onset and then identify the main peak by finding the maximum between two successive detected onsets.

\subsection{Beat-to-Beat Artifact Detection Based on Decision List.} The artifact detection algorithm is a classifier that distinguishes artifacts from reliable pulse waves. We propose the decision list method, which consists of nine decision rules defined by the pulse wave characteristic parameters. If the corresponding rule is not satisfied, it indicates that the pulse wave is disturbed. Before fiducial points detection and artifact detection, we add a block to detect sensor-off and clipping waveform. The template matching method 


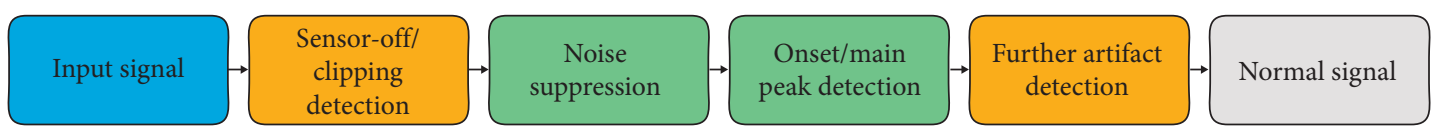

Figure 1: Flowchart of our proposed method. Green boxes indicate fiducial points detection. Yellow boxes indicate artifact detection.

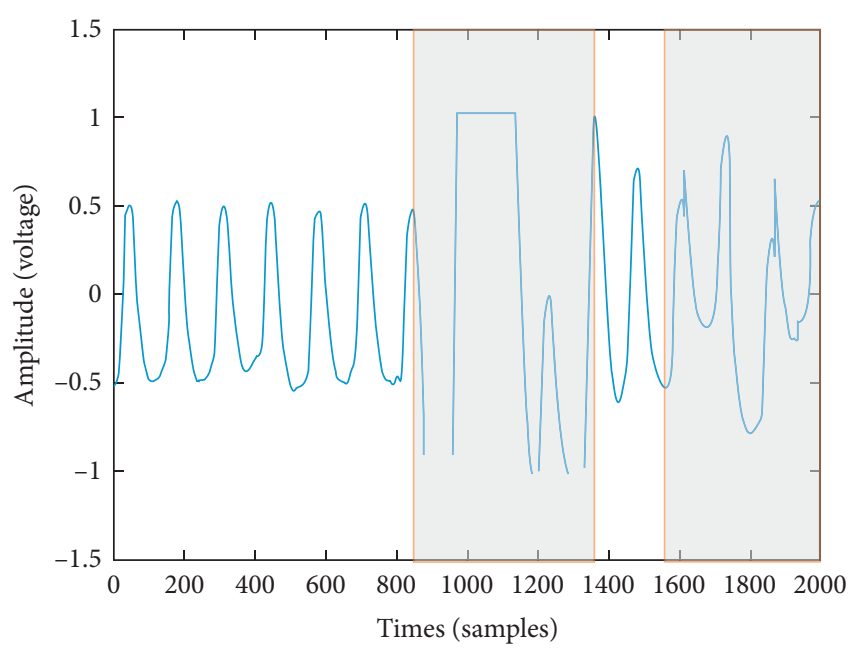

FIGURE 2: Different kinds of artifacts. Grey boxes indicate the sensor-off/clipping waveforms. Green boxes indicate other artifacts.

(template length set to 10 samples here) identifies the signal segments with invariant amplitude.

The basic idea of this method is that each rule can determine whether the current signal is an artifact. The result determined by the current rule cannot be changed by the next rule. As Figure 6 illustrates, rules 1-2 intend to detect jitters or drifts of the signal due to serious noises. The allowable range for pulse wave rise time and pulse wave duration (PWD) is $0.08-0.49 \mathrm{~s}$ [15] and $0.27-2.4 \mathrm{~s}$ [16], respectively (rules 304 ). Moreover, the ratio of systolic and diastolic phase duration is set to 4 (rule 5) according to the experiment. Furthermore, rules 6-9 are based on the Gaussian fitting method we propose.

The amplitude normalization is applied for the singlebeat pulse wave $x(n)$, as shown in the following equation:

$$
x(n)=\frac{x(n)-\max \{x(n)\}}{\max \{x(n)\}-\min \{x(n)\}} .
$$

The pulse waveform consists of two peaks (main peak and dicrotic peak) $[17,18]$. Two Gaussian functions are used to fit the different components of the single-beat pulse wave, and their expressions are as follows:

$$
\begin{aligned}
f(n) & =\sum_{k=1}^{2} g_{k}(n)+x \\
g_{k}(n) & =H_{k} * \exp \left(-\frac{2 *\left(n-C_{k}\right)^{2}}{W_{k}^{2}}\right), \\
J & =\sum_{i=1}^{N}(f(n)-x(n))^{2},
\end{aligned}
$$

TABLE 1: The description of databases, subjects, and signal type used for different algorithms.

\begin{tabular}{lccc}
\hline Database & Subjects & Signal & Algorithm \\
\hline MIMIC & 50 & ABP & Onset detection \\
CSL & 2 & ABP & Peak detection \\
CapnoBase & 33 & PPG & Peak detection \\
MIMIC & 27 & PPG & Artifact detection \\
CSL & 2 & ABP & Artifact detection \\
Local dataset & 10 & PPG & Artifact detection \\
\hline
\end{tabular}

where $n=1,2, \ldots, N, N$ denotes the length of the singlebeat pulse wave, $H, C$, and $W$ denote height, central location, and width of Gaussian function, and $x$ denotes the wandering caused by the noise. $g_{1}(n)$ and $g_{2}(n)$ indicate the main wave and the dicrotic wave. In this paper, after the periodic segmentation, the single-beat signal length is resampled to 1000. Afterward, the upper and lower bounds of the seven fitted parameters $(H 1, C 1, W 1, H 2$, $C 2, W 2$, and $x$ ) are set between $[0.1,0,1,0.1,0,1,0]$ and $[1$, $400,300,0.8,600,400,1]$. The initial values of them are set as $[1,20,15,0.3,55,15,0.2]$. Finally, the objective function $J$ is defined as the sum of squares of errors between the fitting signal $f(n)$ and the normalized signal $x(n)$, as shown in equation (4). Seven parameters are obtained by solving the $J$ based on nonlinear least squares. Rules 6-9 use four parameters ( $H 1, C 1, H 2$, and $C 2)$. According to the relative position of $C 2$ and the end of the pulse wave, as well as the relative position of $C 1$ and $C 2$, the pulse wave is recognized as three patterns. The relative position of $C 1$ and $C 2$ above 35 or the ratio of $H 2$ to $H 1$ above 0.8 is considered abnormal. Figure 7 shows the result of Gaussian functions fitting.

2.4. Assessment Protocol. We assess the performance by applying benchmark parameters: true positive (TP), true negative (TN), false positive (FP), false negative (FN), sensitivity (SE), specificity (SP), positive predictive value (PP), and accuracy (ACC).

If the time difference between the detected point and the reference point is within a predefined acceptable interval (AI), the fiducial point detected by our algorithm is regarded as TP. We set the AI to 1.25 samples for peak detection and two samples for onset detection.

For the artifact detection algorithm, a normal pulse wave is regarded as TP. If continuous artifacts completely cover the original pulse waves, the onset detection algorithm cannot accurately segment the pulse wave and merge them into one artifact. Therefore, the TN number of disturbed signals in such a case can only be estimated by dividing the artifact duration by the average period of two adjacent normal pulse waves. 


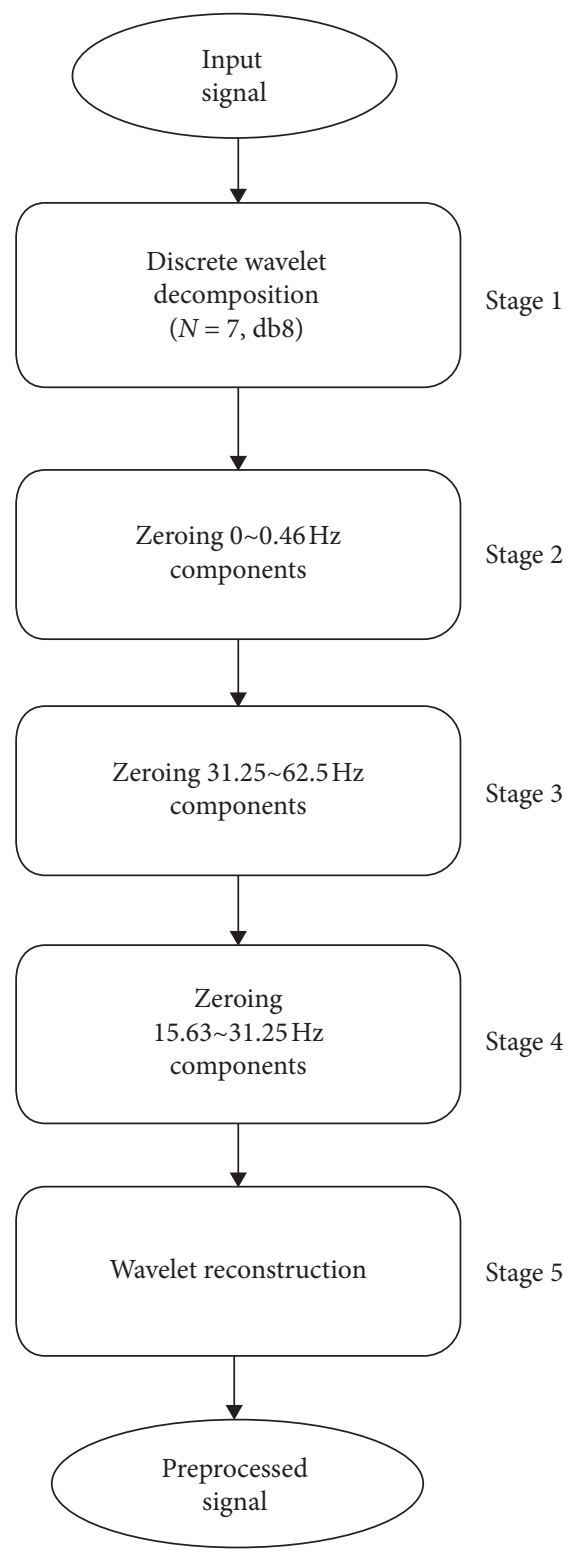

(a)

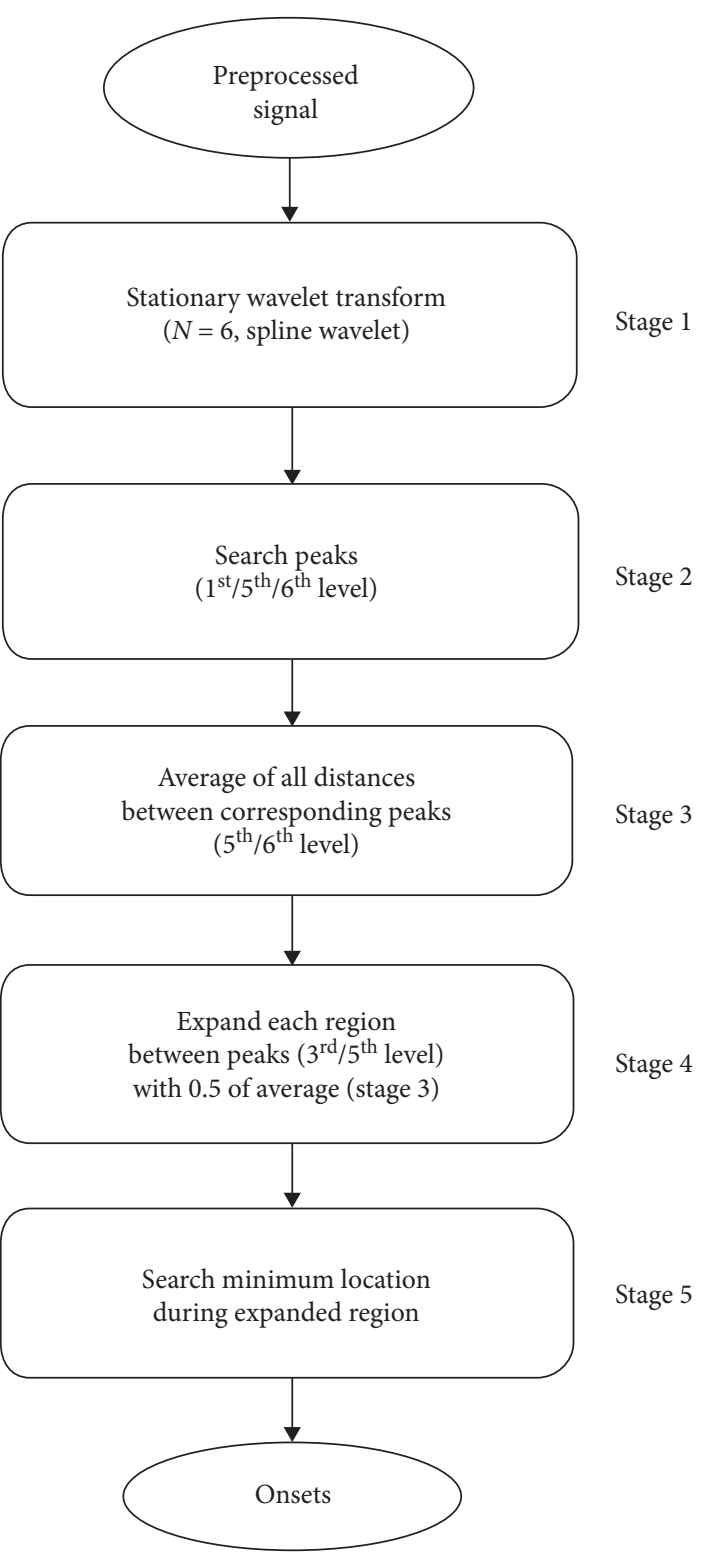

(b)

FIgure 3: Flowchart of the onset detection. (a) Noise suppression based on DWT. (b) Onset detection based on SWT.

\section{Results}

3.1. Fiducial Point Detection. The MIMIC database contains five types of pulse waveforms, as shown in Figure 8. The identical subject can be counted in different kinds according to various types. The results of onset detection are listed in Table 2. The evaluation results show that the proposed method achieved SE ranging from $95.46 \%$ to $98.78 \%$ and PP ranging from $95.89 \%$ to $98.92 \%$ for five types of pulse waveforms.

The results of the main peak detection are summarized in Table 3. Our method is evaluated on a total number of 22,201 peaks from CapnoBase and a total number of 13,115 from CSL. For a fair comparison, we choose the methods based on the CSL database. As Table 4 shows, our approach has a better performance than others.

3.2. Artifact Detection. The performance of beat-to-beat artifact detection was calculated for each database (Table 5). We analyzed 1,460 normal pulse waves and 965 disturbed pulses from the MIMIC database. On the CSL database, a total of 889 normal pulse waves and 144 disturbed pulse waves were analyzed. The algorithm is applied to 523 normal pulse waves and 408 disturbed pulse waves from the local dataset. Figure 9 is an example of beat-to-beat artifact detection. 


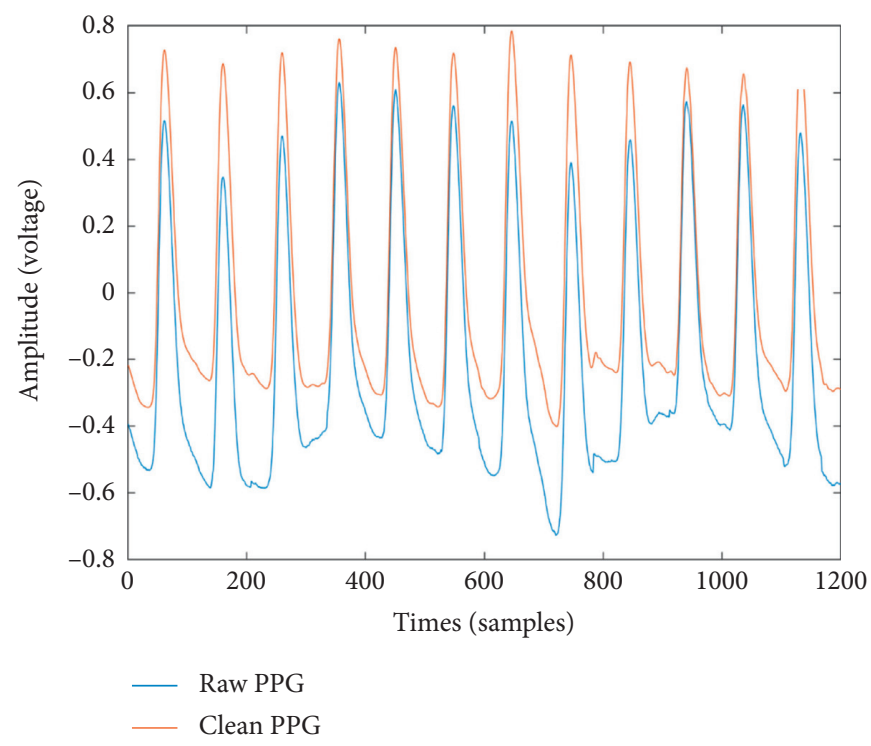

FIgURE 4: The performance of the noise suppression algorithm.
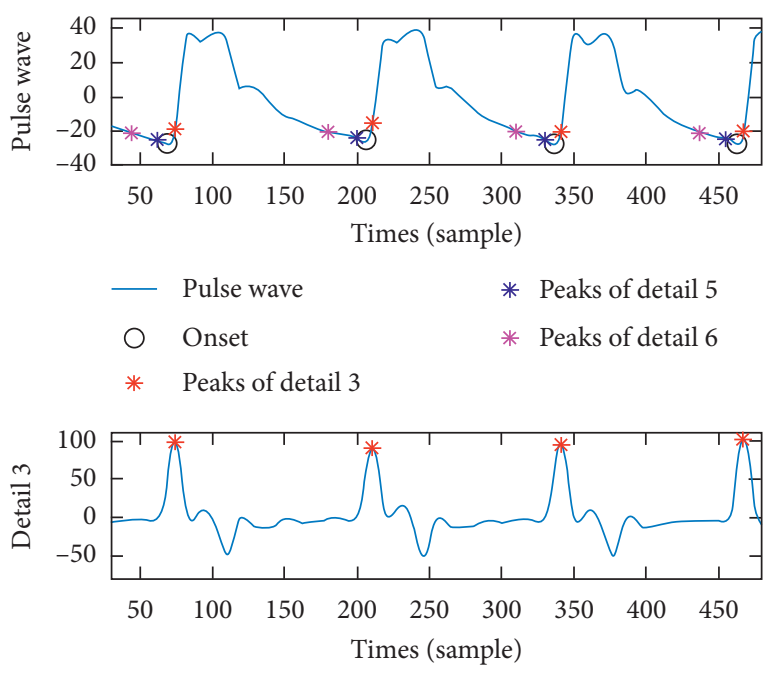

- Detail 3

* Peaks of detail 3

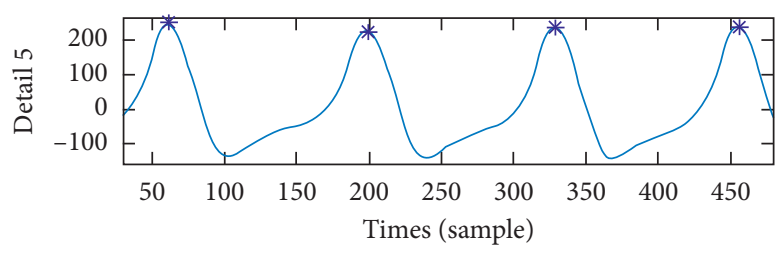

— Detail 5

* Peaks of detail 5

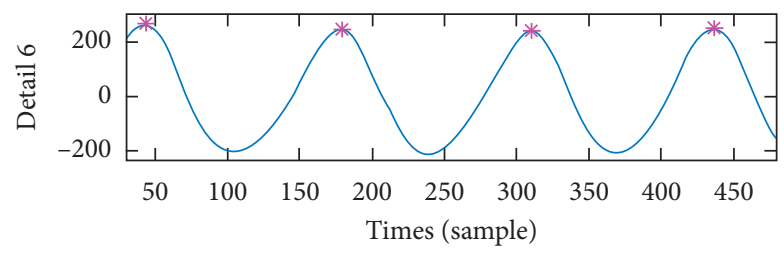

- Detail 6

* Peaks of detail 6

Figure 5: Onset detection based on SWT. Detail 3, detail 5, and detail 6 mean third, fifth, and sixth detail subbands, respectively. Star marks mean peaks on different subbands. The top subfigure means the corresponding positions of subband peaks on the pulse wave. 


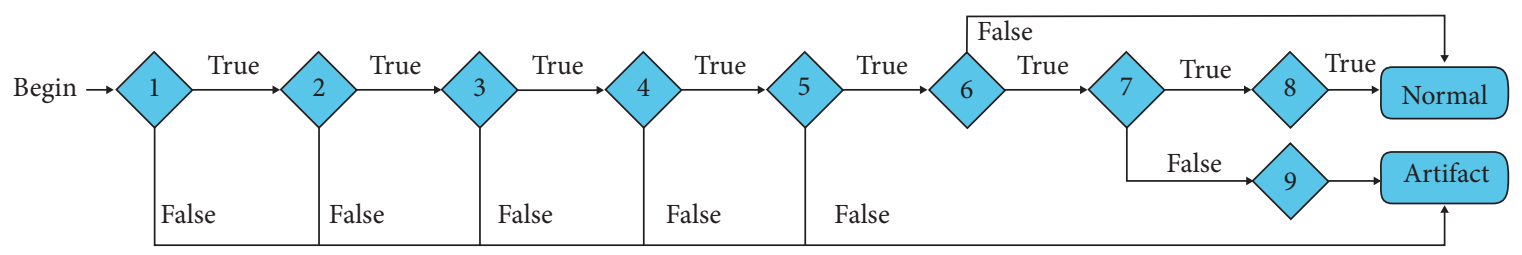

Rule 1: Detect whether the sample is smaller or equal to the sample before during the systolic phase

Rule 5: Systolic phase/diastolic phase $<4$ Rule 2: (Difference of the onset and the next onset)/(pulse wave amplitude) $<0.35$ Rule 3: $0.49 \mathrm{~s}>$ systolic phase $>0.08 \mathrm{~s}$ Rule 6: $C 2<$ The end of the phase wave Rule 7: $35>$ Difference of $C 1$ and $C 2>25$ Rule 8: $H 2 / H 1<0.8$

Rule $4: 2.4 \mathrm{~s}>$ PWD $>0.27 \mathrm{~s}$

Rule 9: Difference of $C 1$ and $C 2>35$

Figure 6: Flowchart of beat-to-beat artifact detection after noise suppression, sensor-off, and clipping detection.
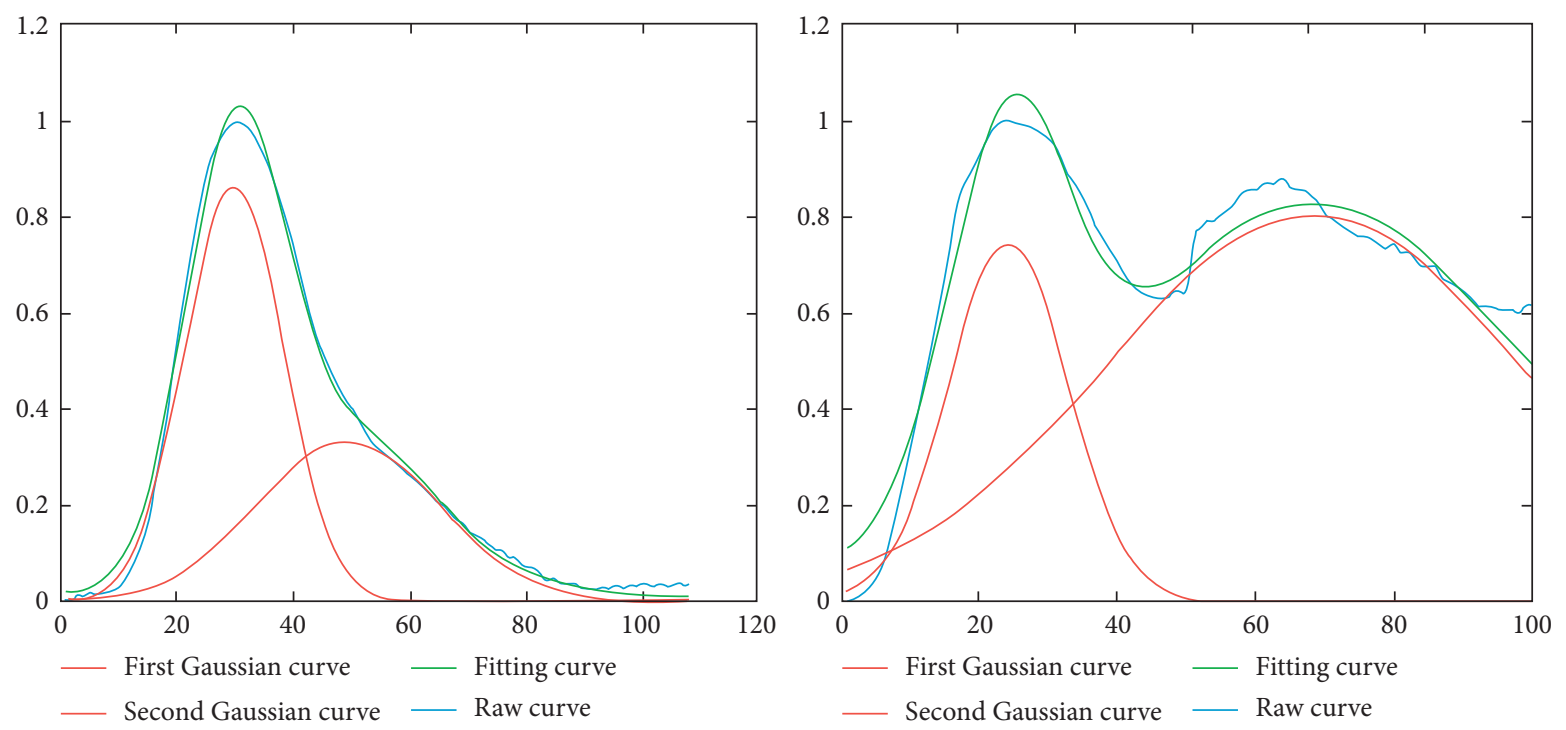

FIgURE 7: Comparison results of Gaussian functions fitting on (a) normal pulse wave and (b) disturbed pulse wave.

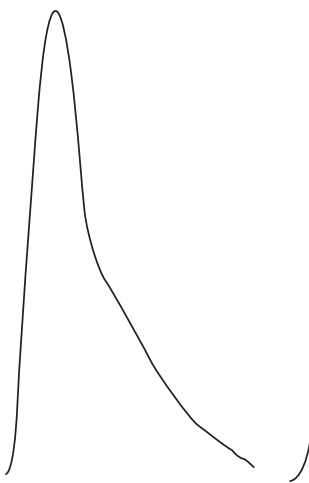

(a)

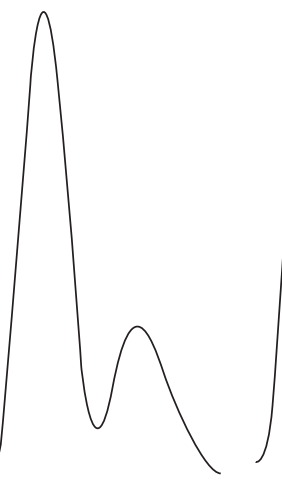

(b)

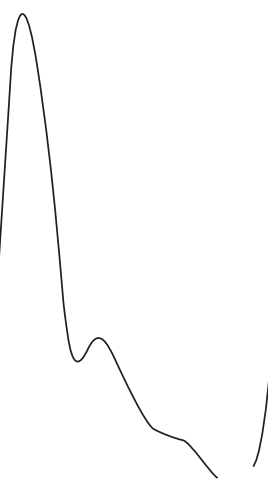

(c)

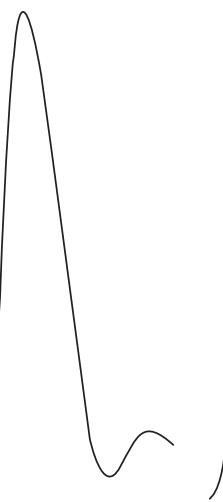

(d)

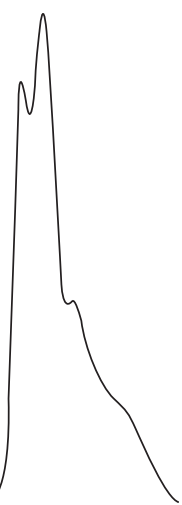

(e)

FIGURE 8: Five types of pulse waveforms: (a) one peak, (b) two peaks (main peak and dicrotic peak), (c) three peaks (main peak, tidal peak, and dicrotic peak), (d) the notch is lower than the onset, and (e) an inflection point appears around the main peak.

\section{Discussion}

The results of peak detection indicate that our method can detect the peaks well although the CapnoBase database and the CSL database both contained artifacts. Since each single- beat pulse wave corresponds to one main peak, the accurate detection of main peaks means the reliable periodic segmentation of the pulse wave. In a word, our method is robust under time-varying pulse wave amplitude, pulse waves of different patterns, and various artifacts and noise. 
TABLE 2: Onset detection performance for pulse waves of different patterns.

\begin{tabular}{lcccccc}
\hline Pattern & $(1)$ & $(2)$ & $(3)$ & $(4)$ & $(5)$ & All \\
\hline Subjects & 15 & 33 & 7 & 3 & 4 & 50 \\
TP & 15707 & 28801 & 5417 & 3218 & 2569 & 42966 \\
FP & 164 & 547 & 229 & 106 & 110 & 819 \\
FN & 186 & 626 & 166 & 153 & 111 & 825 \\
SE (\%) & 98.78 & 97.87 & 97.03 & 95.46 & 95.86 & 98.12 \\
PP (\%) & 98.92 & 98.14 & 95.94 & 96.81 & 95.89 & 98.13 \\
ACC (\%) & 97.73 & 96.09 & 93.20 & 92.55 & 92.08 & 96.31 \\
\hline
\end{tabular}

Table 3: Peak detection performance for different databases.

\begin{tabular}{lccc}
\hline Database & CapnoBase & CSL & All \\
\hline Subjects & 33 & 2 & 35 \\
TP & 21951 & 13049 & 35000 \\
FP & 101 & 36 & 137 \\
FN & 149 & 30 & 179 \\
SE (\%) & 99.33 & 99.77 & 99.49 \\
PP (\%) & 99.54 & 99.72 & 99.61 \\
ACC (\%) & 98.87 & 99.50 & 99.11 \\
\hline
\end{tabular}

TABLE 4: Comparison of peak detection results on the CSL database.

\begin{tabular}{lcccc}
\hline Method & AI (ms) & SE (\%) & PP (\%) & Acc (\%) \\
\hline Fischer [1] & 16 & 99.80 & 99.63 & 99.43 \\
Paradkar [8] & - & 99.24 & 98.48 & 98.41 \\
Iliev [19] & 16 & 99.53 & 99.59 & 98.13 \\
Ours & 10 & 99.77 & 99.72 & 99.50 \\
\hline
\end{tabular}

TABLE 5: The performance of artifact detection for different databases.

\begin{tabular}{lcccc}
\hline & SE $(\%)$ & SP (\%) & PP (\%) & ACC (\%) \\
\hline MIMIC & 99.04 & 97.62 & 98.43 & 97.51 \\
CSL & 100 & 94.44 & 99.17 & 99.11 \\
Local dataset & 98.09 & 95.59 & 96.61 & 94.82 \\
\hline
\end{tabular}

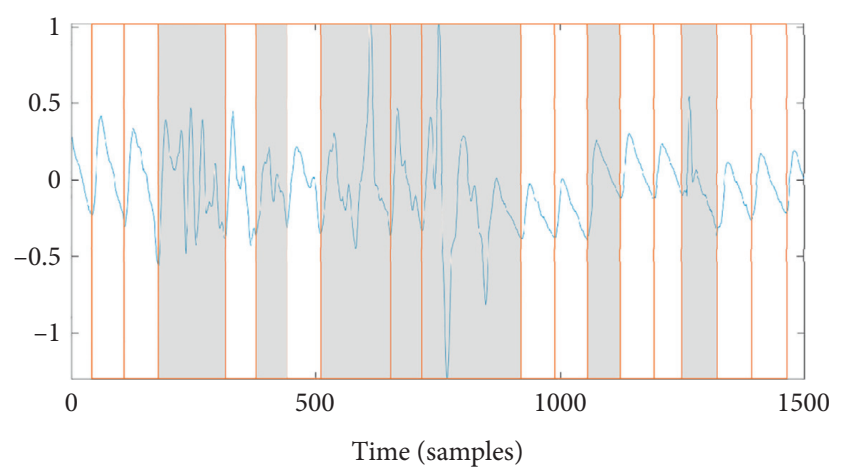

Figure 9: An example of beat-to-beat artifact detection. Filled grey boxes mean artifacts. Yellow vertical lines indicate the segmentation based on the onset detection.

The pulse wave varies in the waveform, making it challenging to calculate the characteristic parameters relying on identifying the dicrotic wave. To solve the above problem, we use two Gaussian functions to fit the main wave and the dicrotic wave of the pulse wave. Besides, our proposed Gaussian fitting method can quantify the changes of two kinds of waves as they propagate in the arteries, which provides another idea for pulse wave analysis. This method estimates the position, height, and width of the dicrotic wave instead of accurate extraction. Gaussian function fitting, combined with characteristic parameters, ensures that pulse waves of different patterns could be analyzed to detect artifacts.

Table 2 presents the onset detection performance of different waveform patterns. The algorithm performance degradation is not due to itself but due to increase in incorrect annotations as waveforms become more complex. Some onsets annotations of the database are not the minimum locations between two peaks. For example, some onsets from the '039' patient are marked on the dicrotic wave.

Although powerful hardware [20] and sophisticated signal processing technique $[21,22]$ can help us convert disturbed signals into original signals, these methods are computationally expensive, and more importantly, they do not take into account the effect of the algorithm on the clean signal. Although some researchers aim to extract heart rate from the disturbed signal without detecting fiducial points and achieve promising results [23], the fiducial point is an important reference. Our method firstly performs periodic segmentation by detecting fiducial points. The longer segmented heart period indicates that the current segment may be an artifact. Subsequent clipping waveform detection and further artifact detection are effective in determining artifacts. In this way, the clean segmented signal is retained as much as possible.

The main limitation of this paper is that the direct performance comparison with other studies is restricted due to the confidentiality of databases and reproducibility of methods. Besides, disease influence on pulse waveform is not taken into account. Besides, the dataset that is used to develop and evaluate our method consists of transmissionmode PPG data, which prevented its application in the device based on the reflectance-mode PPG sensor. So, it is more suitable for the applications in the hospital.

\section{Conclusions}

In this paper, we propose a novel beat-to-beat artifact detection method, which can analyze different kinds of pulse waves to detect artifacts. Our proposed method can be applied to annotate the fiducial points for its robustness. Besides, it can get more effective data segments by eliminating beat-to-beat disturbed signals, improving the calculation of subsequent physiological parameters. Our method is easy to be applied in various mobile devices, which is conducive to promoting smart healthcare and the construction of smart cities.

\section{Data Availability}

The processed data required to reproduce these findings cannot be shared at this time as the data also form part of an ongoing study. 


\section{Conflicts of Interest}

The authors declare that they have no conflicts of interest regarding the publication of this paper.

\section{Authors' Contributions}

Qihan $\mathrm{Hu}$ and Xintao Deng have contributed equally to this work.

\section{Acknowledgments}

This work was supported by the National Natural Science Foundation of China (61071004), Shanghai Science and Technology Support Project (18441900900), Shanghai Municipal Science and Technology Major Project (2017SHZDZX01), and Project of Shanghai Engineering Research Center (19DZ2250800).

\section{References}

[1] C. Fischer, B. Domer, T. Wibmer, and T. Penzel, "An algorithm for real-time pulse waveform segmentation and artifact detection in photoplethysmograms," IEEE Journal of Biomedical and Health Informatics, vol. 21, no. 2, pp. 372-381, 2017.

[2] H. Zhang, Y. Sun, Y. Lu, J. Lan, and Y. Ji, "A novel motion and noise artifacts reduction mechanism (MNARM) for wearable PPG-based heart rate extraction," in Proceedings of the 2015 IET International Conference on Biomedical Image and Signal Processing (ICBISP 2015), pp. 1-4, Beijing, China, November 2015.

[3] J. Lee, M. Kim, H.-K. Park, and I. Y. Kim, "Motion artifact reduction in wearable photoplethysmography based on multichannel sensors with multiple wavelengths," Sensors, vol. 20, no. 5, p. 1493, 2020.

[4] M. S. Islam, M. Shifat-E-Rabbi, A. M. A. Dobaie, and M. K. Hasan, "PREHEAT: precision heart rate monitoring from intense motion artifact corrupted PPG signals using constrained RLS and wavelets," Biomedical Signal Processing and Control, vol. 38, pp. 212-223, 2017.

[5] G. Biagetti, P. Crippa, L. Falaschetti, S. Orcioni, and C. Turchetti, "Motion artifact reduction in photoplethysmography using Bayesian classification for physical exercise identification," in Proceedings of the International Conference on Pattern Recognition Applications and Methods (ICPRAM), pp. 467-474, Rome, Italy, 2016.

[6] D. Dao, S. M. A. Salehizadeh, Y. Noh et al., "A robust motion artifact detection algorithm for accurate detection of heart rates from photoplethysmographic signals using time-frequency spectral features," IEEE Journal of Biomedical and Health Informatics, vol. 21, no. 5, pp. 1242-1253, 2017.

[7] H. Han and J. Kim, "Artifacts in wearable photoplethysmographs during daily life motions and their reduction with least mean square based active noise cancellation method," Computers in Biology and Medicine, vol. 42, no. 4, pp. 387393, 2012.

[8] N. Paradkar and S. R. Chowdhury, "Primary study for detection of arterial blood pressure waveform components," in Proceedings of the 2015 37th Annual International Conference of the IEEE Engineering in Medicine and Biology Society (EMBC), pp. 1959-1962, Milan, Italy, August 2015.

[9] M. Saeed, M. Villarroel, A. T. Reisner et al., "Multiparameter intelligent monitoring in intensive care II: a public-access intensive care unit database*," Critical Care Medicine, vol. 39, no. 5, pp. 952-960, 2011.

[10] M. Aboy, J. McNames, T. Thong, D. Tsunami et al., "An automatic beat detection algorithm for pressure signals," IEEE Transactions on Biomedical Engineering, vol. 52, no. 10, pp. 1662-1670, 2005.

[11] W. Karlen, J. M. Ansermino, and G. Dumont, “Adaptive pulse segmentation and artifact detection in photoplethysmography for mobile applications," in Proceedings of the 2012 Annual International Conference of the IEEE Engineering in Medicine and Biology Society, pp. 3131-3134, San Diego, CA, USA, August 2012.

[12] S. Vadrevu and M. S. Manikandan, "A robust pulse onset and peak detection method for automated PPG signal analysis system," IEEE Transactions on Instrumentation and Measurement, vol. 68, no. 3, pp. 807-817, 2019.

[13] C. Liu, L. Qiao, and G. D. Clifford, "Evaluation of the accuracy and noise response of an open-source pulse onset detection algorithm on pulsatile waveform databases," in Proceedings of the 2016 Computing in Cardiology Conference (CinC), Vancouver, Canada, September 2016.

[14] M. Okada, S. Kimura, and M. Okada, "Estimation of arterial pulse wave velocities in the frequency domain: method and clinical considerations," Medical \& Biological Engineering \& Computing, vol. 24, no. 3, pp. 255-260, 1986.

[15] R. P. Lewis, S. E. Rittogers, W. F. Froester, and H. Boudoulas, "A critical review of the systolic time intervals," Circulation, vol. 56, no. 2, pp. 146-158, 1977.

[16] H. Tanaka, K. D. Monahan, and D. R. Seals, "Age-predicted maximal heart rate revisited," Journal of the American College of Cardiology, vol. 37, no. 1, pp. 153-156, 2001.

[17] K. H. Parker and C. J. H. Jones, "Forward and backward running waves in the arteries: analysis using the method of characteristics," Journal of Biomechanical Engineering, vol. 112, no. 3, pp. 322-326, 1990.

[18] R. Banerjee, A. Ghose, A. R. Choudhury, A. Sinha, and A. Pal, "Noise cleaning and Gaussian modeling of smart phone photoplethysmogram to improve blood pressure estimation," in Proceedings of the International Conference on Acoustics, Speech, and Signal Processing, pp. 967-971, Brisbane, Australia, April 2015.

[19] I. Iliev, B. Nenova, I. Jekova, and V. Krasteva, “Algorithm for real-time pulse wave detection dedicated to non-invasive pulse sensing," in Proceedings of the 2012 Computing in Cardiology, pp. 777-780, Krakow, Poland, September 2012.

[20] P. T. Gibbs, L. Wood, and H. Asada, "Active motion artifact cancellation for wearable health monitoring sensors using collocated mems accelerometers," Smart Structures and Materials, vol. 5765, pp. 811-819, 2005.

[21] K. T. Tanweer, S. R. Hasan, M. Awais, and Kamboh, "Motion artifact reduction from PPG signals during intense exercise using filtered X-LMS," in Proceedings of the 2017 IEEE International Symposium on Circuits and Systems (ISCAS), pp. 1-4, Baltimore, MD, USA, May 2017.

[22] B. Lee, J. Han, H. J. Baek, J. H. Shin, K. S. Park, and W. J. Yi, "Improved elimination of motion artifacts from a photoplethysmographic signal using a Kalman smoother with simultaneous accelerometry," Physiological Measurement, vol. 31, no. 12, pp. 1585-1603, 2010.

[23] H. Chung, H. Lee, and J. Lee, "Finite state machine framework for instantaneous heart rate validation using wearable photoplethysmography during intensive exercise," IEEE Journal of Biomedical and Health Informatics, vol. 23, no. 4, pp. 1595-1606, 2019. 